\title{
An Improved Method to Compute the Inverse Mellin Transform of Holonomic Sequences
}

\author{
Jakob Ablinger*广 \\ Research Institute for Symbolic Computation (RISC) \\ Johannes Kepler University Linz, Altenberger Straße 69, A-4040 Linz, Austria \\ E-mail: jablingeerisc.jku.at
}

We describe a new method to compute the inverse Mellin transform of holonomic sequences, that is based on a method to compute the Mellin transform of holonomic functions. The method is implemented in the computer algebra package HarmonicSums.

Loops and Legs in Quantum Field Theory (LL2018)

29 April 2018 - 04 May 2018

St. Goar, Germany

\footnotetext{
* Speaker.

${ }^{\dagger}$ The research was funded by the Austrian Science Fund (FWF) grant no. SFB F50 (F5009-N15).
} 


\section{Introduction}

There have been several methods proposed to compute the inverse Mellin transform of special sequences, for instance in [1] an algorithm (using rewrite rules) to compute the inverse Mellin transform of harmonic sums was stated. This algorithm was extended in [2] to generalized harmonic sums such as S-sums and cyclotomic sums. A different approach to compute inverse Mellin transforms of binomial sums was described in [3]. In [4] a method to compute the inverse Mellin transform of general holonomic sequences was described. That method uses holonomic closure properties and was implemented in the computer algebra package HarmonicSums $[2,5,6,7,8]$. In the frame of this article we want show how this method can be modified in order to find a more efficient and improved method to compute the inverse Mellin transform of holonomic sequences. The resulting method has been heavily used in the frame of the work on [9].

In the following we repeat important definitions and properties (compare $[3,4,10]$ ). Let $\mathbb{K}$ be a field of characteristic 0. A function $f=f(x)$ is called holonomic (or D-finite) if there exist polynomials $p_{d}(x), p_{d-1}(x), \ldots, p_{0}(x) \in \mathbb{K}[x]$ (not all $p_{i}$ being 0 ) such that the following holonomic differential equation holds:

$$
p_{d}(x) f^{(d)}(x)+\cdots+p_{1}(x) f^{\prime}(x)+p_{0}(x) f(x)=0 .
$$

We emphasize that the class of holonomic functions is rather large due to its closure properties. Namely, if we are given two such differential equations that contain holonomic functions $f(x)$ and $g(x)$ as solutions, one can compute holonomic differential equations that contain $f(x)+g(x)$, $f(x) g(x)$ or $\int_{0}^{x} f(y) d y$ as solutions. In other words any composition of these operations over known holonomic functions $f(x)$ and $g(x)$ is again a holonomic function $h(x)$. In particular, if for the inner building blocks $f(x)$ and $g(x)$ the holonomic differential equations are given, also the holonomic differential equation of $h(x)$ can be computed.

Of special importance is the connection to recurrence relations. A sequence $\left(f_{n}\right)_{n \geq 0}$ with $f_{n} \in \mathbb{K}$ is called holonomic (or P-finite) if there exist polynomials $p_{d}(n), p_{d-1}(n), \ldots, p_{0}(n) \in \mathbb{K}[n]$ (not all $p_{i}$ being 0 ) such that the holonomic recurrence

$$
p_{d}(n) f_{n+d}+\cdots+p_{1}(n) f_{n+1}+p_{0}(n) f_{n}=0
$$

holds for all $n \in \mathbb{N}$ (from a certain point on). In the following we utilize the fact that holonomic functions are precisely the generating functions of holonomic sequences: if $f(x)$ is holonomic, then the coefficients $f_{n}$ of the formal power series expansion

$$
f(x)=\sum_{n=0}^{\infty} f_{n} x^{n}
$$

form a holonomic sequence. Conversely, for a given holonomic sequence $\left(f_{n}\right)_{n \geq 0}$, the function defined by the above sum (i.e., its generating function) is holonomic (this is true in the sense of formal power series, even if the sum has a zero radius of convergence). Note that given a holonomic differential equation for a holonomic function $f(x)$ it is straightforward to construct a holonomic recurrence for the coefficients of its power series expansion. For a recent overview of this holonomic machinery and further literature we refer to [10]. An additional property of 
holonomic functions was given for example in [4] and [3]: if the Mellin transform of a holonomic function

$$
\mathbf{M}[f(x)](n):=\int_{0}^{1} x^{n} f(x) d x=F(n)
$$

is defined, i.e., the integral $\int_{0}^{1} x^{n} f(x) d x$ exists, then it is a holonomic sequence. And using the properties of the Mellin transform we can easily check that

$$
\mathbf{M}\left[x^{m} f^{(p)}(x)\right](n)=\frac{(-1)^{p}(n+m) !}{(n+m-p) !} \mathbf{M}[f(x)](n+m-p)+\sum_{i=0}^{p-1} \frac{(-1)^{i}(n+m) !}{(n+m-i) !} f^{(p-1-i)}(1) .(1
$$

Conversely, if the Mellin transform $\mathbf{M}[f(x)](n)$ of a function $f(x)$ is holonomic, then also the function $f(x)$ is holonomic. In this article we will report on an improved method to calculate the inverse Mellin transform in terms of iterated integrals, note that this method is implemented in the the package HarmonicSums.

The paper is organized as follows. In Section 2 we revisit a method to derive a differential equation for $f(x)$ under the assumption that a holonomic recurrence for $\mathbf{M}[f(x)](n)$ is given. In Section 3 we present a new improved method to compute the inverse Mellin transform of holonomic sequences and finally in Section 4 we give a detailed example to show the application of the method.

\section{Deriving the differential equation}

In this section we want to recall how we can compute a differential equation for $f(x)$ given a holonomic recurrence for $\mathbf{M}[f(x)](n)$ (compare [4]). First we state an important property of the Mellin transform that will be useful in the remainder of this section:

$$
\frac{d^{m}}{d n^{m}} \mathbf{M}[f(x)](n)=\mathbf{M}\left[\log (x)^{m} f(x)\right](n) .
$$

Analyzing (1.4) we see that

$$
\begin{aligned}
\mathbf{M}\left[(-1)^{p} x^{m+p} f^{(p)}(x)\right](n)= & \frac{(n+m+p) !}{(n+m) !} \mathbf{M}[f(x)](n+m) \\
& +\sum_{i=0}^{p-1} \frac{(-1)^{i+p}(n+m+p) !}{(n+m+p-i) !} f^{(p-1-i)}(1) .
\end{aligned}
$$

Hence we get

$$
\begin{aligned}
n^{p} \mathbf{M}[f(x)](n+m)= & \mathbf{M}\left[(-1)^{p} x^{m+p} f^{(p)}(x)\right](n)-a(n) \mathbf{M}[f(x)](n+m) \\
& -\sum_{i=0}^{p-1} \frac{(-1)^{i+p}(n+m+p) !}{(n+m+p-i) !} f^{(p-1-i)}(1),
\end{aligned}
$$

where $a(n) \in \mathbb{K}[n]$ with $\operatorname{deg}(a(n))<p$. We can use this observation to compute the differential equation recursively: Let

$$
p_{d}(n) f_{n+d}+\cdots+p_{1}(n) f_{n+1}+p_{0}(n) f_{n}=0
$$


be the holonomic recurrence for $\mathbf{M}[f(x)](n)$. Let $k:=\max _{0 \leq i \leq d}\left(\operatorname{deg}\left(p_{i}(x)\right)\right)$ and let $c$ be the coefficient of $n^{k}$ in the recurrence i.e.,

$$
c=\sum_{i=0}^{d} c_{i} f_{n+i}
$$

for some $c_{i} \in \mathbb{K}$. For $0 \leq i \leq d$ we replace $c_{i} n^{k} f_{n+i}$ by

$$
c_{i} n^{k} f_{n+i}+\mathbf{M}\left[c_{i}(-1)^{k} x^{k+i} f^{(k)}(x)\right](n)-c_{i}(-1)^{k} \underbrace{\mathbf{M}\left[x^{k+i} f^{(k)}(x)\right](n)}_{*}
$$

and apply (1.4) to $*$. Considering (2.3) we conclude that we reduced the degree of $n$. We apply this strategy until we have removed all appearances of $f_{n+i}$. At this point we deal with an equation of the form

$$
\mathbf{M}\left[q_{l}(x) f^{(l)}(x)+\cdots+q_{1}(x) f^{\prime}(x)+q_{0}(x) f(x)\right](n)+\sum_{j=0}^{k-1} r_{j}(n) f^{(j)}(1)=0
$$

where $r_{i}(n) \in \mathbb{K}[n]$. If all $r_{i}(n)=0$, we can immediately conclude that $f(x)$ has to satisfy the differential equation

$$
f^{(l)}(x)+\cdots+q_{1}(x) f^{\prime}(x)+q_{0}(x) f(x)=0 .
$$

If not all $r_{i}(n)=0$, let $m:=\max _{0 \leq i \leq k-1}\left(\operatorname{deg}\left(r_{j}(n)\right)\right)$, we differentiate equation $(2.5)(m+1)$-times with respect to $n$. According to (2.1) we get

$$
\mathbf{M}\left[\log (x)^{m}\left(q_{l}(x) f^{(l)}(x)+\cdots+q_{1}(x) f^{\prime}(x)+q_{0}(x) f(x)\right)\right](n)=0,
$$

and hence $f(x)$ has to satisfy the differential equation (2.6).

\section{The Inverse Mellin Transform of Holonomic Sequences}

In the following, we deal with the following problem:

Given a nested sum $F(n)$ of the form

$$
F(n):=F_{0}(n) \sum_{i_{1}=1}^{n} F_{1}\left(i_{1}\right) \sum_{i_{2}=1}^{i_{1}} F_{2}\left(i_{2}\right) \cdots \sum_{i_{k}=1}^{i_{k-1}} F_{k}\left(i_{k}\right)
$$

with $F_{j}(n)$ holonomic in $n$ such that $\frac{F_{j}(y)}{F_{j}(y+1)} \in \mathbb{K}(\eta)(y)^{1}$. Note that we could incorporate $\eta$ into the field $\mathbb{K}$, but in our applications $\eta$ represents a specific number with $0<\eta<1$ and we keep $\eta$ explicit since it is important in the regularization later on.

Find, whenever possible, a representation in the form

$$
H(n)=\sum_{j=0}^{k} v_{j}^{n}\left(d_{0, j}+\int_{0}^{1}\left(x^{n}-a_{j}^{n}\right) \sum_{i=1}^{b_{j}} d_{i, j} f_{i, j}(x) d x\right),
$$

\footnotetext{
${ }^{1}$ In this particular instance $F_{j}(y)$ is also called hypergeometric with respect to $y$.
} 
such that $F(n)=H(n)$ for all $n \in \mathbb{N}$ with $n>n_{0}$ for some $n_{0} \in \mathbb{N}$ where in our cases $v_{j}, a_{j}, d_{i, j} \in$ $\mathbb{K}(\eta)$ and $f_{i, j}(x)$ are expressions of the form $p(\eta, x) g(x)$ with $p(\eta, x) \in \mathbb{K}(\eta)(x)$ and $g(x)$ is an iterated integral of the form

$$
\mathrm{G}\left(g_{1}(\tau), g_{2}(\tau), \cdots, g_{k}(\tau), x\right)=\int_{0}^{x} g_{1}\left(\tau_{1}\right) \mathrm{G}\left(g_{2}(\tau), \cdots, g_{k}(\tau), \tau_{1}\right) d \tau_{1},
$$

with $g_{j}(x)$ holonomic in $x$ such that $\frac{g_{j}(y)}{g_{j}^{\prime}(y)} \in \mathbb{K}(\eta)(y)^{2}$ and with the special cases

$$
\mathrm{G}(x)=1
$$

and

$$
\mathrm{G}(\underbrace{\frac{1}{\tau}, \frac{1}{\tau}, \ldots, \frac{1}{\tau}}_{k \text { times }}, x)=\frac{1}{k !} \log (x)^{k} .
$$

In order to find such a representation we start by defining

$$
\bar{F}_{j}(n):=F_{0}(n) \sum_{i_{1}=1}^{n} F_{1}\left(i_{1}\right) \sum_{i_{2}=1}^{i_{1}} F_{2}\left(i_{2}\right) \cdots \sum_{i_{j}=1}^{i_{j-1}} F_{j}\left(i_{j}\right)
$$

for $0 \leq j \leq k$. Hence for example $\bar{F}_{k}(n)=F(n), \bar{F}_{k-1}(n)$ is the original sum with the innermost summation quantifier dropped, $\bar{F}_{1}(n)=F_{0}(n) \sum_{i_{1}=1}^{n} F_{1}\left(i_{1}\right)$ and $\bar{F}_{0}(n)=F_{0}(n)$. Now for each $j$ with $0 \leq j \leq k$ we proceed as follows:

- Determine $v_{j}$, note that $v_{j}^{n}$ reflects the asymptotic behavior of $F_{j}(n)$.

- Construct a recurrence

$$
p_{d}(n) f_{n+d}+\cdots+p_{1}(n) f_{n+1}+p_{0}(n) f_{n}=0,
$$

such that $v_{j}^{-n} \bar{F}_{j}(n)$ is a solution of (3.4).

- Use the method from Section 2 to derive a differential equation

$$
q_{b_{j}}(x) f^{\left(b_{j}\right)}(x)+\cdots+q_{1}(x) f^{\prime}(x)+q_{0}(x) f(x)=0
$$

for the inverse Mellin transform of $v_{j}^{-n} \bar{F}_{j}(n)$.

- Compute, if possible, a general solution ${ }^{3}$

$$
\sum_{i=1}^{b_{j}} d_{i, j} f_{i, j}(x)
$$

of (3.5) in terms of iterated integrals by using the algorithms form [11, 12, 13, 14, 15], see also [16].

\footnotetext{
${ }^{2} g_{j}(y)$ is also called hyperexponential with respect to $y$.

${ }^{3}$ If only a subspace of the general solution can be computed, the result can be still obtained provided that such a representation exists.
} 
- Read off $a_{j} \in(0,1]$ from (3.6), note that $a_{j}$ is a zero of the denominator or 0 .

At this point we use the ansatz

$$
F(n)=\sum_{j=0}^{k} v_{j}^{n}\left(d_{0, j}+\sum_{i=1}^{b_{j}} d_{i, j} \int_{0}^{1}\left(x^{n}-a_{j}^{n}\right) f_{i, j}(x) d x\right),
$$

and it remains to determine the $d_{i, j}$. Therefore, for each $j$ we compute

$$
M_{j}(n)=\sum_{i=1}^{b_{j}} d_{i, j} \int_{0}^{1}\left(x^{n}-a_{j}^{n}\right) f_{i, j}(x) d x,
$$

i.e., we compute the Mellin transform of $\sum_{i=0}^{b_{j}} d_{i, j} f_{i, j}(x)$ for symbolic $d_{i, j}$. Hence we have

$$
F(n)=\sum_{j=0}^{k} v_{j}^{n}\left(d_{0, j}+M_{j}(n)\right),
$$

and by checking a sufficient amount of initial values we can determine the $d_{i, j}$.

\section{A detailed Example}

Let's consider the following expression $(0<\eta<1)$ :

$$
F(n):=4^{-n}\left(\begin{array}{c}
2 n \\
n
\end{array}\right) \sum_{i=1}^{n} \frac{4^{i}\left(\frac{1}{1-\eta}\right)^{i} \sum_{j=1}^{i} \frac{(1-\eta)^{j}}{j}}{i^{2}\left(\begin{array}{c}
2 i \\
i
\end{array}\right)} .
$$

We proceed as suggested in the previous section. Hence we define:

$$
\begin{aligned}
& \bar{F}_{2}(n):=4^{-n}\left(\begin{array}{c}
2 n \\
n
\end{array}\right) \sum_{i=1}^{n} \frac{4^{i}\left(\frac{1}{1-\eta}\right)^{i}}{i^{2}\left(\begin{array}{c}
2 i \\
i
\end{array}\right)} \sum_{j=1}^{i} \frac{(1-\eta)^{j}}{j} \\
& \bar{F}_{1}(n):=4^{-n}\left(\begin{array}{c}
2 n \\
n
\end{array}\right) \sum_{i=1}^{n} \frac{4^{i}\left(\frac{1}{1-\eta}\right)^{i}}{i^{2}\left(\begin{array}{c}
2 i \\
i
\end{array}\right)}, \\
& \bar{F}_{0}(n):=4^{-n}\left(\begin{array}{c}
2 n \\
n
\end{array}\right) .
\end{aligned}
$$

In order to find $v_{0}, v_{1}$ and $v_{2}$ we determine the asymptotic behavior of $\bar{F}_{0}(n), \bar{F}_{1}(n)$ and $\bar{F}_{2}(n)$, respectively. Since,

$$
\underbrace{4^{-n}\left(\begin{array}{c}
2 n \\
n
\end{array}\right)}_{\sim(1)^{n}\left(\frac{1}{\sqrt{\pi}} \frac{1}{\sqrt{n}}+O\left(\frac{1}{n}\right)\right)} \sum_{i=1}^{n} \underbrace{\frac{4^{i}\left(\frac{1}{1-\eta}\right)^{i}}{i^{2}\left(\begin{array}{c}
2 i \\
i
\end{array}\right)}}_{\sim\left(\frac{1}{1-\eta}\right)^{i}\left(\frac{1}{\sqrt{i^{3}}}+O\left(\frac{1}{i^{2}}\right)\right)} \sum_{j=1}^{i} \underbrace{\frac{(1-\eta)^{j}}{j}}_{\sim(1-\eta)^{j}\left(\frac{1}{j}+O\left(\frac{1}{j^{2}}\right)\right)},
$$

we have

$$
\bar{F}_{2}(n) \sim\left(1 \cdot \frac{1}{1-\eta} \cdot(1-\eta)\right)^{n}\left(\frac{\log (\eta)}{\eta^{2} n^{2}}+0\left(\frac{1}{n^{3}}\right)\right)=1^{n}\left(\frac{\log (\eta)}{\eta^{2} n^{2}}+0\left(\frac{1}{n^{3}}\right)\right)(n \rightarrow \infty),
$$




$$
\begin{aligned}
& \bar{F}_{1}(n) \sim\left(1 \cdot \frac{1}{1-\eta}\right)^{n}\left(\frac{1}{\eta} \frac{1}{n^{2}}+0\left(\frac{1}{n^{3}}\right)\right)=\left(\frac{1}{1-\eta}\right)^{n}\left(\frac{1}{\eta} \frac{1}{n^{2}}+0\left(\frac{1}{n^{3}}\right)\right)(n \rightarrow \infty), \\
& \bar{F}_{0}(n) \sim 1^{n}\left(\frac{1}{\sqrt{\pi}} \frac{1}{\sqrt{n}}+O\left(\frac{1}{n}\right)\right)(n \rightarrow \infty),
\end{aligned}
$$

and hence $v_{0}=v_{2}=1$ and $v_{1}=\frac{1}{1-\eta}$.

Now for $j=2$ we derive a recurrence for

$$
v_{2}^{-n} \bar{F}_{2}(n)=4^{-n}\left(\begin{array}{c}
2 n \\
n
\end{array}\right) \sum_{i=1}^{n} \frac{4^{i}\left(\frac{1}{1-\eta}\right)^{i}}{i^{2}\left(\begin{array}{c}
2 i \\
i
\end{array}\right)} \sum_{j=1}^{i} \frac{(1-\eta)^{j}}{j}
$$

and find

$$
\begin{aligned}
0= & (1+n)(2+n)(1+2 n) f(n)+(2+n)\left(-17+6 \eta-20 n+7 \eta n-6 n^{2}+2 \eta n^{2}\right) f(1+n) \\
& +\left(85-61 \eta+104 n-72 \eta n+43 n^{2}-29 \eta n^{2}+6 n^{3}-4 \eta n^{3}\right) f(2+n) \\
& +2(\eta-1)(3+n)^{3} f(3+n) .
\end{aligned}
$$

We compute a differential equation for the inverse Mellin transform of $v_{2}^{-n} \bar{F}_{2}(n)$ using the method presented in Section 1 and get

$$
\begin{aligned}
0= & 2(\eta-1) f(x)+(11-14 x-3 \eta+14 x \eta) f^{\prime}(x) \\
& +\left(-5+17 x-12 x^{2}-7 x \eta+12 x^{2} \eta\right) f^{\prime \prime}(x)+2 x(1-x+x \eta) f^{(3)}(x) .
\end{aligned}
$$

with the general solution $d_{1,2} f_{1,2}(x)+d_{2,2} f_{2,2}(x)+d_{3,2} f_{3,2}(x)$ with $d_{1,2}, d_{2,2}, d_{3,2} \in \mathbb{K}(\eta)$ and

$$
\begin{aligned}
f_{1,2}(x)= & \frac{1}{\sqrt{1-x} \sqrt{x}}, \\
f_{2,2}(x)= & \frac{\sqrt{1-x} \sqrt{x}-2 \sqrt{1-x} x^{3 / 2}+4 \mathrm{G}(\sqrt{1-\tau} \sqrt{\tau}, x)}{\sqrt{1-x} \sqrt{x}}, \\
f_{3,2}(x)= & \frac{2 \mathrm{G}(\sqrt{1-\tau} \sqrt{\tau}, x)}{(1-\eta) \sqrt{1-x} \sqrt{x}}-\frac{\mathrm{G}\left(\frac{1}{1-\tau+\eta \tau}, x\right)}{1-\eta}+\frac{\eta \mathrm{G}\left(\frac{1}{1-\tau+\eta \tau}, x\right)}{1-\eta}+\frac{2 x \mathrm{G}\left(\frac{1}{1-\tau+\eta \tau}, x\right)}{1-\eta} \\
& -\frac{2 \eta x \mathrm{G}\left(\frac{1}{1-\tau+\eta \tau}, x\right)}{1-\eta}-\frac{\mathrm{G}\left(\frac{\sqrt{1-\tau} \sqrt{\tau}}{1-\tau+\eta \tau}, x\right)}{(1-\eta) \sqrt{1-x} \sqrt{x}}-\frac{\eta \mathrm{G}\left(\frac{\sqrt{1-\tau} \sqrt{\tau}}{1-\tau+\eta \tau}, x\right)}{(1-\eta) \sqrt{1-x} \sqrt{x}} \\
& -\frac{4 \mathrm{G}\left(\sqrt{1-\tau} \sqrt{\tau}, \frac{1}{1-\tau+\eta \tau}, x\right)}{(1-\eta) \sqrt{1-x} \sqrt{x}}+\frac{4 \eta \mathrm{G}\left(\sqrt{1-\tau} \sqrt{\tau}, \frac{1}{1-\tau+\eta \tau}, x\right)}{(1-\eta) \sqrt{1-x} \sqrt{x}} .
\end{aligned}
$$

Since the solution has no pole in $0<x<1$ we can set $a_{2}=0$.

Next for $j=1$ derive a recurrence for

$$
v_{1}^{-n} \bar{F}_{1}(n)=(1-\eta)^{n} 4^{-n}\left(\begin{array}{c}
2 n \\
n
\end{array}\right) \sum_{i=1}^{n} \frac{4^{i}\left(\frac{1}{1-\eta}\right)^{i}}{i^{2}\left(\begin{array}{c}
2 i \\
i
\end{array}\right)},
$$

and find

$$
0=-(\eta-1)(1+n)(1+2 n) f(n)+\left(-8+6 \eta-11 n+7 \eta n-4 n^{2}+2 \eta n^{2}\right) f(1+n)
$$




$$
+2(2+n)^{2} f(2+n)
$$

Now we compute a differential equation and get

$$
0=2 f(x)+3(-1+\eta+2 x) f^{\prime}(x)+2 x(-1+\eta+x) f^{\prime \prime}(x),
$$

for which we find the solution $d_{1,1} f_{1,1}(x)+d_{2,1} f_{2,1}(x)$ with $d_{1,1}, d_{2,1} \in \mathbb{K}(\eta)$ and

$$
\begin{aligned}
f_{1,1}(x) & =\frac{1}{\sqrt{1-\eta-x} \sqrt{x}}, \\
f_{2,1}(x) & =\frac{\left(\sqrt{1-\eta-x} \sqrt{x}-\eta \sqrt{1-\eta-x} \sqrt{x}-2 \sqrt{1-\eta-x} x^{3 / 2}+4 \mathrm{G}(\sqrt{1-\eta-\tau} \sqrt{\tau}, x)\right)}{(1-\eta)^{2} \sqrt{1-\eta-x} \sqrt{x}} .
\end{aligned}
$$

Again, since the solution has no pole in $0<x<1$ we can set $a_{1}=0$.

Finally for $j=0$ we derive a recurrence for

$$
v_{0}^{-n} \bar{F}_{0}(n)=4^{-n}\left(\begin{array}{c}
2 n \\
n
\end{array}\right),
$$

and find

$$
0=(-1-2 n) f(n)+2(1+n) f(1+n) .
$$

We compute a differential equation and get

$$
0=(-1+2 x) f(x)+2(x-1) x f^{\prime}(x)=0,
$$

which has the solution $d_{1,0} f_{1,0}(x)$ with $d_{1,0} \in \mathbb{K}(\eta)$ and

$$
f_{1,0}(x)=\frac{1}{\sqrt{1-x} \sqrt{x}} .
$$

Since the solution has no pole in $0<x<1$ we again set $a_{0}=0$. Since $v_{0}=v_{2}, a_{0}=a_{2}$ and $f_{1,0}(x)=f_{1,2}(x)$ we don't have to consider this solution separately.

Summarizing we have

$$
\begin{aligned}
F(n)= & \sum_{j=1}^{2} v_{j}^{n}\left(d_{0, j}+\sum_{i=1}^{b_{j}} d_{i, j} \int_{0}^{1}\left(x^{n}-a_{j}^{n}\right) f_{i, j}(x) d x\right) \\
= & d_{0,2}+d_{1,2} \int_{0}^{1} x^{n} f_{1,2}(x) d x+d_{2,2} \int_{0}^{1} x^{n} f_{2,2}(x) d x+d_{3,2} \int_{0}^{1} x^{n} f_{3,2}(x) d x \\
& +\frac{1}{(1-\eta)^{n}}\left(d_{0,1}+d_{1,1} \int_{0}^{1} x^{n} f_{1,1}(x) d x+d_{2,1} \int_{0}^{1} x^{n} f_{2,1}(x) d x\right) .
\end{aligned}
$$

And it remains to fix the $d_{i, j}$. Therefore we compute the Mellin transforms and get

$$
\begin{aligned}
F(n)= & d_{0,2}+d_{1,2}\left(-\frac{2 \sqrt{\pi}}{4^{n}}\left(\begin{array}{c}
2 n \\
n
\end{array}\right)\right)+d_{2,2}\left(-\frac{1}{4^{n}}\left(\begin{array}{c}
2 n \\
n
\end{array}\right) \sum_{i=1}^{n} \frac{4^{i}}{\left(\begin{array}{c}
2 i \\
i
\end{array}\right) i^{2}}+\cdots\right) \\
& +d_{3,2}\left(-\frac{\left(\begin{array}{c}
2 n \\
n
\end{array}\right)}{(1-\eta) 4^{n}} \sum_{i=1}^{n} \frac{(1-\eta)^{i} \sum_{j=1}^{i} \frac{4^{j}}{\left(\begin{array}{c}
2 j \\
j
\end{array}\right)(1-\eta)^{j} j^{2}}}{i}+\cdots\right)+d_{0,1} \frac{1}{(1-\eta)^{n}}
\end{aligned}
$$




$$
+d_{1,1}\left(\frac{-\sqrt{\eta}\left(\begin{array}{c}
2 n \\
n
\end{array}\right)}{4^{n}} \sum_{i=1}^{n} \frac{4^{i}}{\left(\begin{array}{c}
2 i \\
i
\end{array}\right)(1-\eta)^{i} i}+\cdots\right)+d_{2,1}\left(\frac{-\left(\begin{array}{c}
2 n \\
n
\end{array}\right)}{4^{n}} \sum_{i=1}^{n} \frac{4^{i}}{\left(\begin{array}{c}
2 i \\
i
\end{array}\right)(1-\eta)^{i} i^{2}}+\cdots\right) .
$$

Now it is straightforward to determine the $d_{i, j}$ by checking initial values and we find

$$
\begin{aligned}
& F(n)=\int_{0}^{1} x^{n} \frac{1}{12 \pi \sqrt{1-x} \sqrt{x}(1-\eta)^{3}}\left(3 \pi^{2}(1-\eta)^{3}-4\left(1+11 \eta+11 \eta^{2}+\eta^{3}\right)\right. \\
& -192 \sqrt{-\eta}(1+\eta) \mathrm{G}(\sqrt{1-\eta-\tau} \sqrt{\tau}, 1) \mathrm{G}\left(\frac{1}{1-\tau+\eta \tau}, 1\right) \\
& +384 \mathrm{G}(\sqrt{1-\eta-\tau} \sqrt{\tau}, 1)^{2} \mathrm{G}\left(\frac{1}{1-\tau+\eta \tau}, 1\right) \\
& -24 \pi(1-\eta)^{3}(1+\eta) \mathrm{G}\left(\frac{\sqrt{1-\tau} \sqrt{\tau}}{1-\tau+\eta \tau}, 1\right) \\
& +96 \pi(1-\eta)^{4} \mathrm{G}\left(\frac{1}{1-\tau+\eta \tau}, \sqrt{1-\tau} \sqrt{\tau}, 1\right) \\
& +192(1-\eta)^{3}(1+\eta) \mathrm{G}\left(\frac{\sqrt{1-\tau} \sqrt{\tau}}{1-\tau+\eta \tau}, \sqrt{1-\tau} \sqrt{\tau}, 1\right) \\
& \left.-768(1-\eta)^{4} \mathrm{G}\left(\frac{1}{1-\tau+\eta \tau}, \sqrt{1-\tau} \sqrt{\tau}, \sqrt{1-\tau} \sqrt{\tau}, 1\right)\right) d x \\
& +\int_{0}^{1} x^{n} \frac{2(1-\eta)\left(-\sqrt{1-x} \sqrt{x}+2 \sqrt{1-x} x^{3 / 2}-4 \mathrm{G}(\sqrt{1-\tau} \sqrt{\tau}, x)\right) \mathrm{G}\left(\frac{1}{1-\tau+\eta \tau}, 1\right)}{\sqrt{1-x} \sqrt{x}} d x \\
& +\int_{0}^{1} x^{n}\left(-\frac{4 \mathrm{G}(\sqrt{1-\tau} \sqrt{\tau}, x)}{\sqrt{1-x} \sqrt{x}}+\frac{2(1+\eta) \mathrm{G}\left(\frac{\sqrt{1-\tau} \sqrt{\tau}}{1-\tau+\eta \tau}, x\right)}{\sqrt{1-x} \sqrt{x}}\right. \\
& +\frac{2\left(\sqrt{1-x} \sqrt{x}-2 \sqrt{1-x} x^{3 / 2}-\sqrt{1-x} \sqrt{x} \eta+2 \sqrt{1-x} x^{3 / 2} \eta\right) \mathrm{G}\left(\frac{1}{1-\tau+\eta \tau}, x\right)}{\sqrt{1-x} \sqrt{x}} \\
& \left.+\frac{2(4-4 \eta) \mathrm{G}\left(\sqrt{1-\tau} \sqrt{\tau}, \frac{1}{1-\tau+\eta \tau}, x\right)}{\sqrt{1-x} \sqrt{x}}\right) d x \\
& +\frac{1}{(1-\eta)^{n}}\left(\int_{0}^{1} x^{n} \frac{2\left(\eta+\eta^{2}+4 \sqrt{-\eta} \mathrm{G}(\sqrt{1-\eta-\tau} \sqrt{\tau}, 1)\right) \mathrm{G}\left(\frac{1}{1-\tau+\eta \tau}, 1\right)}{\sqrt{x} \sqrt{1-x-\eta}(-1+\eta) \sqrt{-\eta}} d x\right. \\
& +\int_{0}^{1} x^{n} \frac{2 \mathrm{G}\left(\frac{1}{1-\tau+\eta \tau}, 1\right)}{\sqrt{x} \sqrt{1-x-\eta}(-1+\eta)}\left(-\sqrt{x} \sqrt{1-x-\eta}+2 x^{3 / 2} \sqrt{1-x-\eta}\right. \\
& +\sqrt{x} \sqrt{1-x-\eta} \eta-4 \mathrm{G}(\sqrt{1-\eta-\tau} \sqrt{\tau}, x)) d x) \text {. }
\end{aligned}
$$

\section{Acknowledgments}

I want to thank J. Blümlein, K. Schönwald and C. Schneider for useful discussions. 


\section{References}

[1] E. Remiddi and J.A.M. Vermaseren, Harmonic Polylogarithms, Int. J. Mod. Phys. A15, 2000. [arXiv:hep-ph/9905237v1]

[2] J. Ablinger, Computer Algebra Algorithms for Special Functions in Particle Physics, PhD Thesis, J. Kepler University Linz, 2012. arXiv:1305.0687 [math-ph].

[3] J. Ablinger, J. Blümlein, C. G. Raab and C. Schneider, Iterated Binomial Sums and their Associated Iterated Integrals, J. Math. Phys. 55 (2014) 112301 doi:10.1063/1.4900836 [arXiv:1407.1822 [hep-th]].

[4] J. Ablinger, Inverse Mellin Transform of Holonomic Sequences, PoS LL 2016 (2016) 067 [arXiv:1606.02845 [cs.SC]].

[5] J. Ablinger, The package HarmonicSums: Computer Algebra and Analytic aspects of Nested Sums, PoS LL 2014 (2014) 019 [arXiv:1407.6180 [cs.SC]].

[6] J. Ablinger, J. Blümlein and C. Schneider, Harmonic Sums and Polylogarithms Generated by Cyclotomic Polynomials, J. Math. Phys. 52 (2011) 102301 doi:10.1063/1.3629472 [arXiv:1105.6063 [math-ph]].

[7] J. Ablinger, J. Blümlein and C. Schneider, Analytic and Algorithmic Aspects of Generalized Harmonic Sums and Polylogarithms, J. Math. Phys. 54 (2013) 082301 doi:10.1063/1.4811117 [arXiv:1302.0378 [math-ph]].

[8] J. Ablinger, A Computer Algebra Toolbox for Harmonic Sums Related to Particle Physics, arXiv:1011.1176 [math-ph].

[9] J. Ablinger, J. Blümlein, A. De Freitas, C. Schneider and K. Schönwald The two-mass contribution to the three-loop pure singlet operator matrix element, Nucl. Phys. B. 927 (2018) [arXiv:1711.06717 [hep-ph]].

[10] M. Kauers and P. Paule, The Concrete Tetrahedron, Text and Monographs in Symbolic Computation, Springer, Wien, 2011.

[11] J.J. Kovacic, An algorithm for solving second order linear homogeneous differential equations, J. Symbolic Comput. 2, 1986.

[12] M. Petkovšek, Hypergeometric solutions of linear recurrences with polynomial coefficients, J. Symbolic Comput. 14, 1992.

[13] S.A. Abramov and M. Petkovšek, D’Alembertian solutions of linear differential and difference equations, in proceedings of ISSAC'94, ACM Press, 1994.

[14] M. Bronstein, Linear Ordinary Differential Equations: breaking through the order 2 barrier, in proceedings of ISSAC'92, ACM Press, 1992.

[15] P.A. Hendriks and M.F. Singer, Solving difference equations in finite terms, J. Symbolic Comput. 27, 1999.

[16] J. Ablinger, Computing the Inverse Mellin Transform of Holonomic Sequences using Kovacic's Algorithm, PoS RADCOR2017 (2017) 069 [arXiv:1801.01039 [cs.SC]]. 